\title{
Efeito do diodo emissor de luz (led) sobre a força de preensão palmar
}

\author{
Sergio Antonio Sapienza* , Richard Eloin Liebano**
}

Resumo: A preensão palmar é um parâmetro do estado de força geral do indivíduo. A terapia a LED altera o conteúdo de ATP nas fibras musculares. A acupuntura libera grandes quantidades de ATP. A luz incoerente também pode ser conduzida pelos meridianos de acupuntura. Assim, o objetivo deste estudo foi avaliar o efeito do LED em pontos de acupuntura sobre a força de preensão palmar. Para tanto, 45 indivíduos foram distribuídos ao acaso em três grupos: G1 cuja força de preensão palmar foi medida antes e após à aplicação do LED, G2 como grupo placebo, e G3 como grupo de controle. Foi utilizado um dinamômetro Jamar ${ }^{\circledR}$ na mensuração da força e um aparelho emissor de LED vermelho. A significância entre os desvios percentuais das forças dos três grupos foi $p=0,395$. Conclui-se que não existe efeito do LED em pontos de acupuntura sobre a força de preensão em indivíduos saudáveis.

Descritores: Força da Mão, Fototerapia, Luz, Terapia por Acupuntura, Terapia pela Cor.

\section{Effect of light-emitting diode (led) on hand grip strength}

Abstract: The hand grip is a parameter of the state of overall strength of individual. The LED therapy alters the content of ATP in muscle fibers. The acupuncture releases large amounts of ATP. The incoherent light can also be conducted by the acupuncture meridians. So, the objective of this study was to evaluate the effect of LED into acupuncture points on the hand grip force. Therefore, 45 subjects were divided into three groups: $\mathrm{G} 1$ group which hand grip force was measured before and after LED application, G2 like placebo group, and G3 like control group. It was used a Jamar ${ }^{\circledR}$ dynamometer to measure the strength and a red LED transmitting unit. The significance among the percentage variation of the forces of the three groups was $p=0,395$. It was concluded that there is no LED effect when applied to acupuncture points on the hand grip force in healthy subjects.

Descriptors: Hand Strength, Phototherapy, Light, Acupuncture Therapy, Color Therapy. 


\section{Introdução}

A principal meta da reabilitação física consiste no tratamento e reeducação de distúrbios que se relacionam com o movimento funcional humano. ${ }^{1} \mathrm{~A}$ mão é considerada um dos principais instrumentos do corpo humano, sendo que boa parte do desenvolvimento da humanidade pode ser creditado a ela, especialmente, pela peculiar característica de possibilitar movimentos de preensão. ${ }^{2}$

A avaliação da força de preensão manual (FPM) do tipo palmar é frequentemente utilizada, especialmente, no âmbito fisioterápico e desportivo. ${ }^{2}$ Isto provavelmente ocorre porque a avaliação da força é um dos aspectos da função manual mais fácil de mensurar de uma maneira objetiva. Assim, a preensão palmar pode ser considerada como um dos parâmetros do estado de força geral dos indivíduos, ${ }^{3}$ e sua diminuição pode ocorrer após vários traumatismos da mão, que envolvem músculos, além de lesões dos nervos periféricos. $^{1}$

Estudos têm demonstrado efeitos significativos do laser visível, luz coerente (luz de mínima divergência e máximo paralelismo ${ }^{4}$ ), como fonte de irradiação sobre as atividades musculares. No entanto, os efeitos da irradiação de diodos emissores de luz (LEDs), fonte de luz incoerente, não foram muito pesquisados. ${ }^{4,5} \mathrm{O}$ efeito da terapia a diodos emissores de luz (LEDT) sobre o desempenho muscular é ligeiramente menor do que com a terapia a laser de baixo nível (LLLT). Porém, isto não representa uma verdadeira diferença entre as duas terapias, porque são necessários estudos mais aprofundados da dosagem das fontes emissoras para esta diferenciação. ${ }^{6}$

Contudo, a utilização de diferentes LEDs de vários comprimentos de onda forneceu pistas sobre seus benefícios como, por exemplo, o efetivo restabelecimento da citocromo $\mathrm{c}$ oxidase para os níveis de controle. A enzima endógena mitocondrial, citocromo c oxidase, é um fotoreceptor biológico importante que intervém na fotobiomodulação no intervalo de luz que vai do vermelho extremo ao infravermelho próximo (NIR). O conteúdo de adenosina trifosfato (ATP) em neurônios primários sobe ou desce em conjunto com a atividade da citocromo $\mathrm{c}$ oxidase, conhecida como enzima geradora de energia. ${ }^{7,8}$ Além disto, a terapia a diodos emissores de luz induz um aumento da citocromo c oxidase nas fibras musculares e é eficaz na melhoria da capacidade aeróbia de fibras musculares intermediárias e vermelhas. Desta forma, o LED pode intervir na melhora do desempenho de humanos saudáveis e apoiar os esforços de reabilitação nas condições em que a contração muscular e movimentos estão comprometidos. ${ }^{9}$

A extra-síntese de ATP em mitocôndrias isoladas e células intactas de vários tipos, sob irradiação com luz de diferentes comprimentos de onda, está bem documentada. O ATP é um combustível universal dentro de células vivas que dirige todas as reações biológicas. Sabese que mesmo pequenas alterações no nível de ATP podem alterar significativamente 0 metabolismo celular ${ }^{10} \mathrm{e}$ a contração muscular depende da energia fornecida pelo ATP. ${ }^{11}$

Além da fototerapia, a acupuntura, que é uma técnica terapêutica cujas origens escritas na China datam mais de 2000 anos, é utilizada para tratar doenças ${ }^{12} \mathrm{e}$ tem demonstrado liberar grandes quantidades de ATP nos queratinócitos, fibroblastos e outras células da

Saúde (Santa Maria), v.39, n.1, p. 139-148, 2013. Efeito do diodo emisso de luz (LED) sobre a força de preensão palmar pele. ${ }^{13}$ No entanto, não foram encontrados até o momento estudos que investigassem esse efeito nos tecidos musculares. Para tanto, desenvolvimento tecnológico tem introduzido novas formas de estimular os pontos de acupuntura. $O$ uso terapêutico da acupuntura a laser 
ganhou rápida popularidade e obtém resultados evidentes no tratamento de vários distúrbios. ${ }^{14,12}$ Porém, existe a preocupação que lasers de baixa potência utilizados por um período prolongado, podem danificar os pontos de acupuntura levando à redução de sua eficácia. ${ }^{4}$

Estudos ${ }^{4}$ apontam que, a luz coerente é convertida no corpo em luz incoerente. Esta luz incoerente é igualmente eficaz na produção de resultados clínicos ${ }^{4}$ e pode ser conduzida através do corpo humano pelos meridianos de acupuntura. ${ }^{15}$ Recentemente, constatou-se que em certos casos a terapia a LEDs promoveu melhores resultados nos processos metabólicos teciduais do que os obtidos com a terapia a laser. ${ }^{5}$

Diante desse contexto, este estudo justifica-se pela escassez de pesquisas sobre a aplicação de fototerapia em pontos de acupuntura para reabilitação fisioterápica, mais especificamente, para o aumento de força muscular.

O objetivo deste estudo é avaliar o efeito do diodo emissor de luz (LED) em pontos de acupuntura, na força de preensão palmar em indivíduos saudáveis, assintomáticos.

\section{Materiais e Métodos}

Este estudo trata-se de um ensaio clínico controlado, aleatorizado e simples-cego.

Indivíduos

Participaram parte deste estudo 45 indivíduos de ambos os sexos, distribuídos ao acaso em três grupos de 15 pessoas cada, que foram recrutados voluntariamente. Todos os participantes assinaram um termo de consentimento livre e esclarecido.

Critério de inclusão

Os seguintes critérios de inclusão foram utilizados: indivíduos de ambos os sexos com idade acima de 17 anos, pois a maior parte dos estudos encontrados relata pesquisas a partir de 18 anos de idade.

Critério de exclusão

Os seguintes critérios de exclusão foram utilizados: qualquer problema musculoesquelético ou doença em membros superiores, problemas cardíacos ou respiratórios, estado de fraqueza ou mal-estar no momento da avaliação que comprometa a mensuração da força de preensão palmar, conforme declarado por eles mesmos no termo de consentimento livre e esclarecido.

Procedimento de randomização e simples-cego

Os participantes foram divididos aleatoriamente em três grupos, G1, G2 e G3, contendo 15 indivíduos cada grupo, com representantes de ambos os sexos. O grupo G1 representou os indivíduos que tiveram a força de preensão palmar avaliada e posteriormente submetidos à aplicação do LED, com nova avaliação de força após a aplicação. Os indivíduos do grupo G2 foram do grupo placebo, que inicialmente tiveram suas forças de preensão palmar medidas e posteriormente, foi supostamente aplicado o LED, porém, sem o aparelho estar ligado, e novamente foram avaliadas as forças. $\mathrm{O}$ grupo $\mathrm{G} 3$ ou grupo controle foi somente

Rev. Saúde (Santa Maria), Santa Maria, v.39, n.1, p. 139-148, Jan./Jul.2013.

Sapienza, S. et al. 
submetido à mensuração da força de preensão palmar por duas vezes, com um intervalo de seis minutos entre ambas, sem qualquer outra intervenção. Os indivíduos dos grupos $\mathrm{G} 1 \mathrm{e}$ G2 foram avaliados com os olhos vendados de forma a não saberem a qual grupo pertenciam.

Materiais

Dinamômetro Jamar ${ }^{\circledR}$

0 dinamômetro Jamar ${ }^{\circledR}$ tem sido descrito na literatura internacional como 0 mais eficiente na mensuração da força de preensão palmar e é o sistema de calibração que ganhou mais aceitação clínica na deteç̧ão desta força, sendo utilizado regularmente em estudos de pacientes. A força de preensão palmar, mensurada com o uso do dinamômetro Jamar $^{\circledR}$, consiste em um procedimento objetivo, prático e de fácil utilização. 0 dinamômetro Jamar $^{\circledR}$ é recomendado pela Sociedade Americana de Terapeutas de Mão (SATM). ${ }^{3}$

Diodo Emissor de Luz (LED)

Foi utilizado um equipamento de LED vermelho com quatro saídas, que permitiu a fixação dos LEDs nos pontos de aplicação através de fita hipoalergênica micropore da marca $3 \mathrm{M}^{\circledR}$, de tal forma que os quatro pontos foram irradiados simultaneamente. Cada LED vermelho possuía comprimento de onda de $625 \mathrm{~nm}$, diâmetro de $3 \mathrm{~mm}$, potência de $0,242 \mathrm{~W} / \mathrm{cm} 2$ e energia de $1,026 \mathrm{~J} /$ ponto.

Procedimento de mensuração da força de preensão palmar

Antes do início do teste foi explicada de forma objetiva aos participantes a sua finalidade. Os materiais foram mostrados e então foi realizada uma medida prévia para familiarização e adaptação ao esquema de teste, que foi realizado na mão dominante do examinado. Durante a avaliação da força de preensão palmar o participante permaneceu sentado, estando o ombro na posição neutra, cotovelo em $90^{\circ}$ e punho na posição neutra intermediária entre pronação e supinação, enquanto o dinamômetro ficou apoiado ou sustentado pelo examinador, seguindo a recomendação da Sociedade Americana de Terapeutas de Mão (SATM), conforme a Figura 1. Foi dado um comando verbal pelo examinador que consistiu na pronúncia da seguinte frase "um, dois, três e já". Utilizou-se a média de três repetições (com duração de 5 segundos para cada tentativa) com a manopla colocada na posição dois (indicada no aparelho). 0 intervalo de tempo entre uma tentativa e outra, para a mesma mão, foi de 1 minuto, a fim de controlar a fadiga muscular durante 0 teste..$^{1,2,3}$

Saúde (Santa Maria), v.39, n.1, p. 139-148, 2013. Efeito do diodo emisso de luz (LED) sobre a força de preensão palmar 


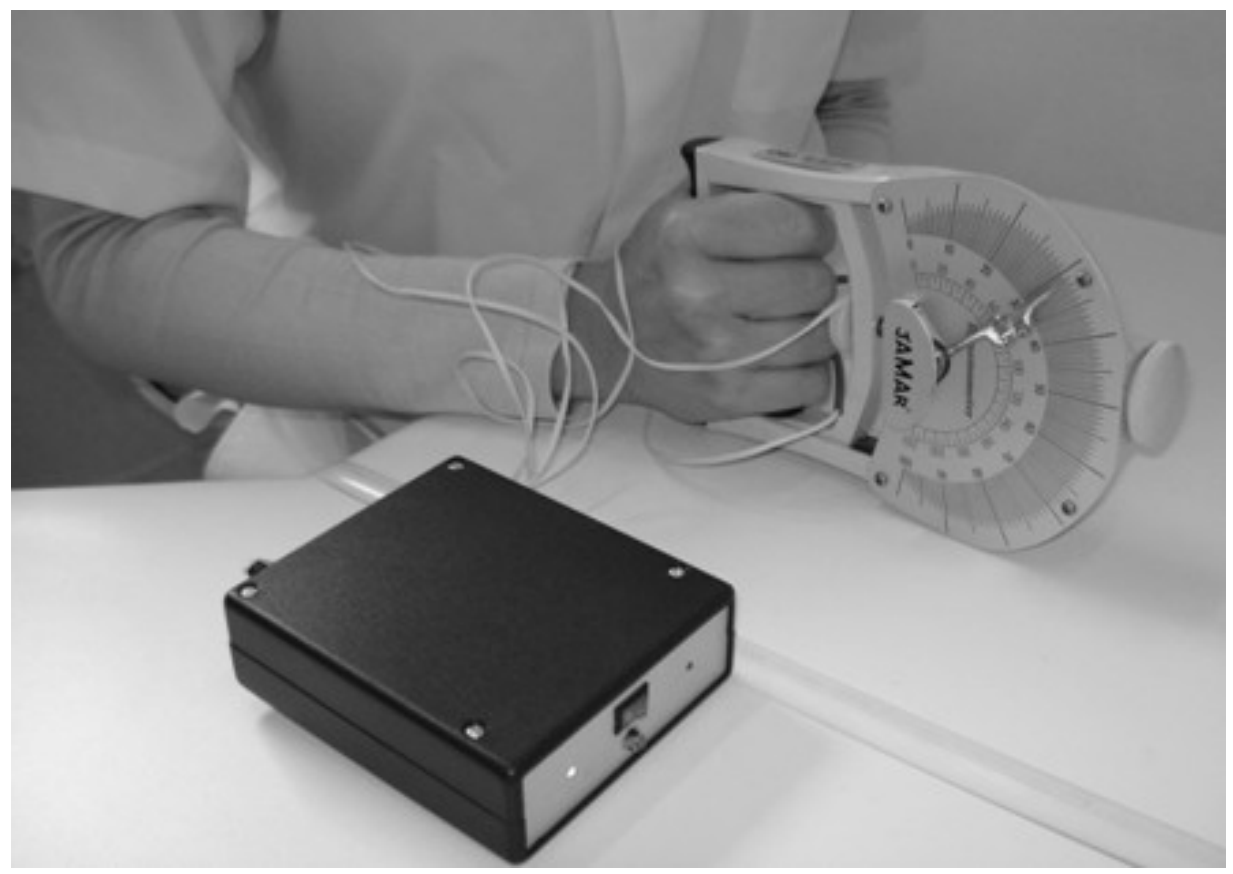

Figura 1 - Mensuração da força de preensão palmar com LEDs fixados.

\section{Aplicação do LED}

Os LEDs vermelhos foram fixados com fita hipoalergênica em quatro pontos de canais de acupuntura cujos trajetos estão relacionados aos principais músculos utilizados na preensão palmar, e ficaram posicionados perpendicularmente à superfície da pele. Os canais e pontos de acupuntura utilizados foram do Pulmão (pontos P11 - Shaoshang e P9 - Taiyuan), do Pericárdio (ponto PC9 - Zhongchong) e do Coração (ponto C9 - Shaochong), conforme mostrado na Figura 2. O tempo de irradiação foi de um minuto sobre os quatro pontos simultaneamente. Esperou-se cinco minutos após a irradiação para que fosse novamente medida a força de preensão palmar.

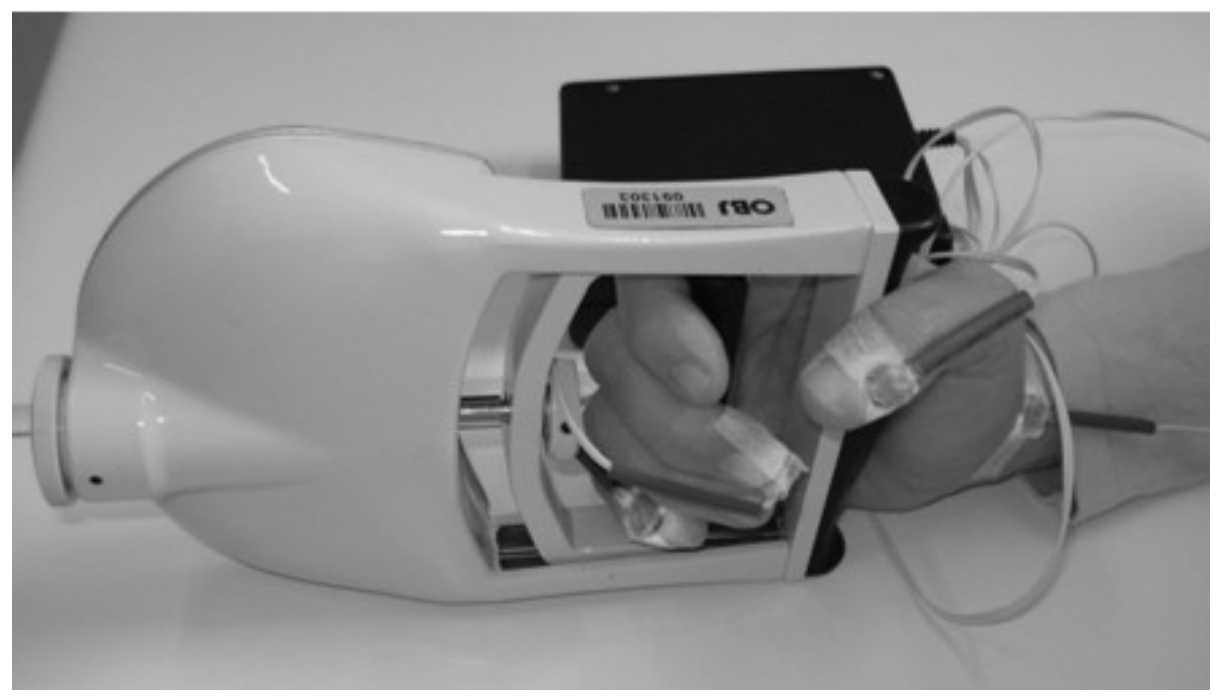

Figura 2 - Fixação dos quatro LEDs em posição de teste.

Rev. Saúde (Santa Maria), Santa Maria, v.39, n.1, p. 139-148, Jan./Jul.2013 Sapienza, S. et al. 
Análise estatística

A normalidade dos dados foi avaliada pelo teste de Shapiro-Wilk que consiste em testar a hipótese nula de que a mostra veio de uma população normalmente distribuída. Para comparação do percentual de alteração da força de preensão palmar entre os grupos utilizouse a Análise de Variância (ANOVA). Os dados foram analisados levando-se em conta suas médias aritméticas e desvios padrões. 0 nível de significância adotado foi de $5 \%(p<0,05)$.

\section{Resultados}

Os 45 indivíduos avaliados, 13 homens e 32 mulheres, possuíam idade média de $36 \pm$ 15 anos.

A Tabela 1 mostra os resultados das forças de preensão palmar médias para cada um dos três grupos e relacionam a variação percentual entre a medição inicial e final com seus respectivos desvios padrões.

Tabela 1 - Forças de preensão palmar inicial e final (média \pm DP).
\begin{tabular}{cccc}
\hline Grupos & $\begin{array}{c}\text { Força média inicial } \\
(\text { Kgf })\end{array}$ & $\begin{array}{c}\text { Força média final } \\
(\text { Kgf })\end{array}$ & $\begin{array}{c}\text { Variação } \\
\text { percentual }(\%)\end{array}$ \\
\hline G1 & $30,9 \pm 11,9$ & $31,6 \pm 11,8$ & $2,8 \pm 10,7$ \\
G2 & $32,6 \pm 13,0$ & $32,8 \pm 12,1$ & $2,2 \pm 8,9$ \\
G3 & $33,8 \pm 13,0$ & $33,0 \pm 12,0$ & $-1,7 \pm 8,1$ \\
\hline
\end{tabular}

Notas: G1 = grupo que recebeu a aplicação do LED, G2 = grupo placebo, G3 = grupo controle.

$\mathrm{Na}$ Figura 3 podem-se observar os valores médios e os limites máximos e mínimos da variação percentual para cada grupo.

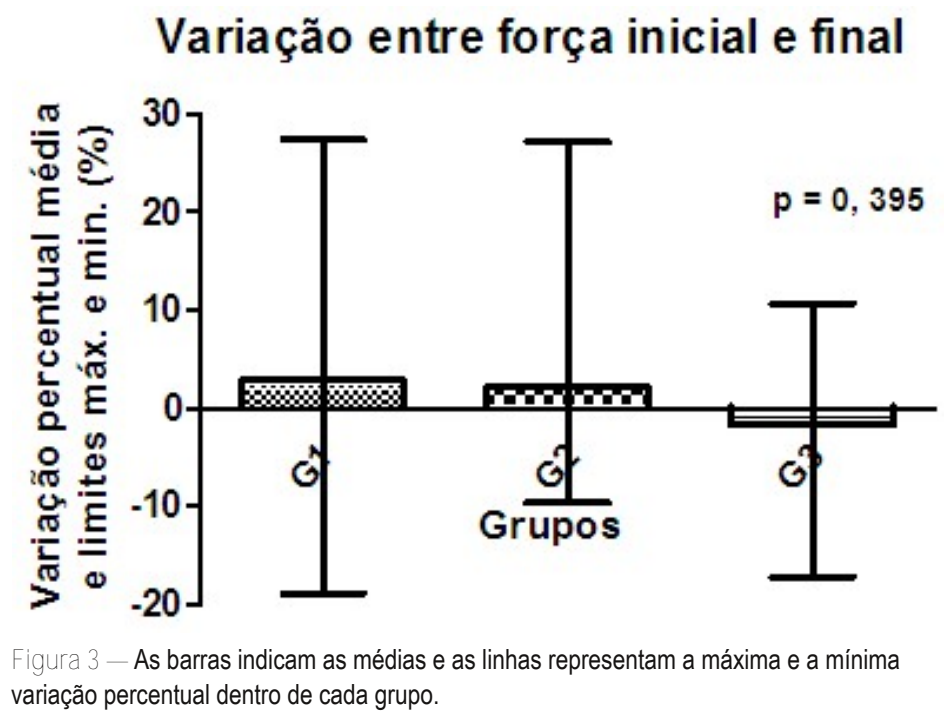

Saúde (Santa Maria), v.39, n.1, p. 139-148, 2013. Efeito do diodo emisso de luz (LED) sobre a força de preensão palmar 


\section{Discussão}

Para a medição da força de preensão palmar, sugere-se que seja padronizado o horário de avaliação e 0 ajuste da empunhadura, levando em consideração o sexo e 0 tamanho da mão do avaliado. Igualmente, com base na literatura, dinamômetros extensiométricos que permitem a aquisição de curvas de força versus tempo podem fornecer uma gama maior de variáveis para análise em comparação a dinamômetros que permitem apenas a medida de força máxima. ${ }^{2} \mathrm{~A}$ disponibilidade dos indivíduos e do aparelho utilizado para a medição não possibilitou a aplicação destas recomendações sugeridas.

Contudo, o dinamômetro Jamar ${ }^{\circledR}$ tem sido considerado o instrumento mais aceito para avaliar a força de preensão palmar desde 1954. Apesar das recomendações estabelecidas pela Sociedade Americana de Terapeutas da Mão que foram utilizadas neste experimento, observa-se que ainda não existe um protocolo comum durante o procedimento de teste com 0 dinamômetro Jamar ${ }^{\circledR}$. 3

Estudos populacionais (com o uso do dinamômetro Jamar ${ }^{\circledR}$ ) realizados no Brasil em indivíduos com faixa etária compreendida entre 20 e 59 anos sugerem que a média geral de força de preensão palmar dos homens, lado dominante, é de 44,2 kgf e entre mulheres, lado dominante é de $31,6 \mathrm{kgf.}^{1}$ Outro estudo brasileiro de 2008 estratifica valores de força de preensão para cada faixa etária e sexo. Entre 20 e 44 anos, para homens 49,35 \pm 7,36 kgf e para mulheres $31,37 \pm 5,82 \mathrm{kgf}$. Entre 45 e 64 anos, para homens $43,28 \pm 7,11 \mathrm{kgf}$ e para mulheres $23,50 \pm 5,35 \mathrm{kgf}^{16}$

Nos grupos G1, G2 e G3, foram observados valores de forças de preensão palmar dentro e fora dos limites de base dos referidos estudos. Em ambos os casos, houve tanto uma variação percentual positiva (aumento de força) quanto negativa (diminuição de força) entre a medição inicial e final da força de preensão palmar. Ou seja, em todos os grupos, não podemos afirmar que há uma relação entre o aumento ou diminuição de força com o valor numérico desta.

As radiações visíveis são fortemente absorvidas pela hemoglobina e melanina (cromóforos). A luz vermelha é absorvida pelos citocromos nas mitocôndrias das células. Todas as células têm esses citocromos, de modo que todos podem ser estimulados pela luz vermelha. Isso por sua vez, possivelmente afeta a permeabilidade da membrana das células. ${ }^{17}$

As radiações vermelhas visíveis têm profundidade de penetração de aproximadamente 1 ou $2 \mathrm{~mm}$. Porém, tem sido mostrada uma associação interessante usando atividade eletromiográfica entre a visualização da luz vermelha e uma maior contração voluntária máxima (força de preenssão). Especula-se que a luz vermelha leva à estimulação e o uso de vermelho no ambiente de tratamento pode aumentar o tônus muscular. ${ }^{17}$

O sistema de cromoterapia Dinshah se encaixa muito bem com a teoria da medicina tradicional oriental, cores relativas a órgãos internos e sistema de meridianos. 0 vermelho é quente e estimulante. As cores quentes como o vermelho, fortalecem e tonificam. ${ }^{4}$ Observa-se que a luz viaja bem pelo corpo apenas quando irradiada nos pontos de acupuntura e 0 vermelho é a segunda cor de maior penetração, vindo após o branco. ${ }^{15}$

O movimento de preensão palmar provoca intensa atividade dos músculos flexor superficial e profundo dos dedos, dos interósseos e do $4^{\circ}$ lumbrical, bem como, nota-se atividade de músculos que realizam o movimento de contrapressão realizado pelo polegar

Rev. Saúde (Santa Maria), Santa Maria, v.39, n.1, p. 139-148, Jan./Jul.2013 Sapienza, S. et al. ISSN $2236-5843$ 
pelo do músculo flexor longo do polegar, de músculos tênares (oponente do polegar, adutor do polegar e flexor curto do polegar) e hipotênares (flexor curto do dedo mínimo), estes agindo como agonistas e contraindo-se isotonicamente. ${ }^{3}$

Os meridianos (canais) de acupuntura chamados de tendino-musculares circulam na periferia do corpo, não penetram os órgãos e vísceras, mas estão associados e possuem os mesmos nomes dos doze canais primários. Todos se originam nas extremidades e sobem para a cabeça e tronco. Acompanham o curso de seu canal primário associado, mas são mais largos, mais superficiais, e acompanham as linhas dos grandes músculos e grupos musculares, tendões e ligamentos. 0 meridiano tendino-muscular do Pulmão tem origem no polegar no ponto Shaoshang (P11) e dirige-se para a eminência tenar, segue o pulso radial e sobe pela área flexora no antebraço até a região central do cotovelo. 0 meridiano tendinomuscular do Pericárdio se origina na ponta do dedo médio, no ponto Zhongchong (PC9), paralelamente ao meridiano tendino-muscular do Pulmão no lado medial do cotovelo, segue pelo lado ântero-medial do antebraço. 0 meridiano tendino-muscular do Coração se origina no lado radial do dedo mínimo, no ponto Shaochong (C9) e se liga ao osso pisiforme do pulso e sobe para pela face medial do cotovelo no antebraço. 18,19,20

Pelo fato dos indivíduos serem saudáveis e assintomáticos, foi utilizada parcialmente a técnica de tratamento de meridianos tendino-musculares que consistiu em estimular os pontos Ting (pontos de origem) e circular a energia dos canais primários (principais) através dos pontos de tonificação. Estes dois pontos são coincidentes para os canais do Coração, Shaochong (C9), e Pericárdio, Zhongchong (PC9). No caso do canal do Pulmão, são os pontos Shaoshang (P11) e Taiyuan (P9), respectivamente. ${ }^{19,20,21}$ Porém, não foi observado no grupo $G 1$, no qual o LED foi aplicado, um aumento significativo e representativo de força após a aplicação do LED. De acordo com a significância encontrada entre os desvios percentuais dos grupos $(p=0,395)$, não houve evidência de diferença significativa entre as médias dos 3 grupos.

Assim, é possível observar que a irradiação muscular através de LED vermelho (640nm), com 1,044J/ponto, aumenta a atividade muscular sem mudar o tempo para fadiga e força. ${ }^{5}$ Em contrapartida, estudos apontam que o efeito da terapia a diodos emissores de luz (LEDT) pode retardar o desenvolvimento de fadiga muscular periférica durante o exercício, utilizando LED vermelho de comprimento de onda de $660 \mathrm{~nm} .{ }^{6} \mathrm{O}$ tempo necessário para que haja um aumento do tamanho de vasos após irradiação de LED vermelho é de cinco minutos após a sua aplicação, e cessa após 10 minutos. ${ }^{5}$

\section{Conclusão}

A partir do objetivo desse estudo, pode-se concluir que não existe efeito do diodo emissor de luz (LED), aplicado em pontos de acupuntura, sobre a força de preensão palmar em indivíduos saudáveis e assintomáticos.

Sugere-se que mais estudos sejam efetuados com irradiação de LEDs com diferentes parâmetros de comprimentos de ondas e energia sobre pontos de canais de acupuntura.

Saúde (Santa Maria), v.39, n.1, p. 139-148, 2013. 


\section{Referências Bibliográficas}

1. Moreira D, Raimundo AKS. Guia prático de testes especiais do aparelho locomotor. Brasilia: Thesaurus; 2005.

2. Dias JA, Ovando AC, Külkamp W, Borges Junior NG. Força de preensão palmar: métodos de avaliação e fatores que influenciam a medida. Rev Bras Cineantropom Desempenho Hum. 2010;12(3):209-16.

3. Moreira D, Alvarez RRA, Gogoy JR, Cambraia AN. Abordagem sobre preensão palmar utilizando 0 dinamômetro JAMAR ${ }^{\circledR}$ : uma revisão de literatura. Rev Bras Ciênc Mov. 2003;11(2):95-9.

4. Cocilovo A. Colored light therapy: overview of its history, theory, recent developments and clinical applications combined with acupuncture. Am J Acupunct. 1999;27(1-2):71-83.

5. Kelencz CA, Muñoz IS, Amorim CF, Nicolau RA. Effect of low-power gallium-aluminum-arsenium noncoherent light $(640 \mathrm{~nm})$ on muscle activity: a clinical study. Photomed Laser Surg. 2010, Oct; 28(5): 64752.

6. Leal Junior EC, Lopes-Martins RA, Rossi RP, De Marchi T, Baroni BM, de Godoi V, Marcos RL, Ramos L, Bjordal JM. Effect of cluster multi-diode light emitting diode therapy (LEDT) on exercise-induced skeletal muscle fatigue and skeletal muscle recovery in humans. Lasers Surg Med. 2009 Oct;41(8):572-7.

7. Desmet KD, Paz DA, Corry JJ, Eells JT, Wong-Riley MT, Henry MM, Buchmann EV, et al. Clinical and Experimental Applications of NIR-LED Photobiomodulation. Photomed Laser Surg. 2006 Apr; 24(2):121-8.

8. Wong-Riley MT, Liang HL, Eells JT, Chance B, Henry MM, Buchmann E, Kane M, Whelan HT. Photobiomodulation directly benefits primary neurons functionally inactivated by toxins: role of cytochrome $\mathrm{C}$ oxidase. J Biol Chem. 2005 Feb 11; 280(6):4761-71.

9. Hayworth CR, Rojas JC, Padilla E, Holmes GM, Sheridan EC, Gonzalez-Lima F. In vivo low-level light therapy increases cytochrome oxidase in skeletal muscle. Photochem Photobiol. 2010 May-Jun; 86(3):67380.

10. Karu T. Mitochondrial Mechanisms of Photobiomodulation in Context of New Data About Multiple Roles of ATP. Photomed Laser Surg. 2010 Apr;28(2):159-60.

11. Guyton CG, Hall JE. Tratado de Fisiologia Médica. 10ª ed. Rio de Janeiro: Guanabara Koogan; 2002.

12. Whittaker P. Laser acupuncture: past, present and future. Lasers Med Sci. 2004;19:69-80.

13. Burmstock G. Acupuncture: a novel hypothesis for the involvement of purinergic signaling. Med Hypoth. 2009;73:470-2.

14. Naeser MA. Introduction to Laser Acupuncture and its Clinical Application. Spinal Cord Injury Medicine 16th Educational Symposium, Naeser Lecture Notes. 2007, Nov.

15. Pankratov S. Meridians conduct light. Raum und Zeit [Internet]. 1991 [acesso em 2011 Mar 26]. Translated from the German by Wolfgang Mitschrich. Disponivel em: http://www.photonstimulator.com/Article\%20Russian.htm.

16. Moura PMLS. Estudo da força de preensão palmar em diferentes faixas etárias do desenvolvimento humano [dissertação]. Brasília: Universidade de Brasília; 2008.

17. Low J., Reed A. Eletroterapia explicada: princípios e prática. $3^{\mathrm{a}}$ ed. São Paulo: Manole; 2001. p. 377-98.

18. Deadman P, Al-Khafaji M, Baker K. A Manual of Acupuncture [CD-ROM]. Howe, East Sussex: Journal of Chinese Medicine Publications; 2000.

19. Yamamura Y, Gentil D, Lozano SA, Yabuta MM, D’Ávila MV. Algias do sistema músculo-esquelético e os canais de energia tendino-musculares. Rev Paul Acupunt. 1999;5(1):1-6.

Rev. Saúde (Santa Maria), Santa Maria, v.39, n.1, p.

139-148, Jan./Jul.2013

Sapienza, S. et al.

ISSN 2236-5843 
20. WHO, World Health Organization. WHO Standard Acupuncture Point Locations in the Western Pacific Region. WHO Library Cataloguing in Publication Data. 2009.

21. Kwang WT. Algias Periféricas. Apostila do Centro de Estudos de Acupuntura e Terapias Alternativas [Internet]. 2010 Mar 8 [acesso em 2011 Nov 10]. Disponível em: http://ceata.com.br/ensino/file.php/2/apostilas_acupuntura/Aula_14/Dores_Superficiais.pdf.

\section{Sergio Antonio Sapienza}

Endereço para correspondência - Rua Inacio Manuel Alvares, $n^{\circ}$ 80, apartamento.43-B. Bairro Rio Pequeno, CEP: 05372-110, São Paulo, SP, Brasil.

E-mail: sergio.sapienza@hotmail.com

Recebido em 04 de julho de 2012.

Aprovado em 17 de janeiro de 2013. 